\title{
Prostatectomía convencional versus resección endoscópica de próstata monopolar en pacientes con Hiperplasia Prostá- tica: un estudio de corte retrospectivo
}

Walter David Egas Ortega ${ }^{1}$, Julio Alberto Luzuriaga Graf ${ }^{1}$, Oscar Hipólito Sánchez Chávez† ${ }^{1}$, Walter Enrique Egas Romero ${ }^{1}$, Luis Andrés Cando Gaibor ${ }^{2}$, Eder Chiriboga Dorado ${ }^{3}$

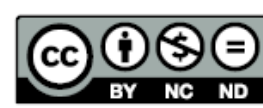
te artículo está bajo na licencia de Creative Commons de tipo ReconoOPEN ACCESS cimiento - No comercial - Sin obras derivadas 4.0 International

1 Hospital Luis Vernaza

2 Hospital Básico del Carmen, Manab

3 Centro de Salud Nicaragua, Vinces, Los Ríos

\section{ORCID ID:}

Walter Egas Ortega Md

https:/ / orcid.org/0000-0002-7539-2323

Julio Alberto Luzuriaga Graff Md.

https://orcid.org/0000-0002-7214-210X

Walter Egas Romero Md.

https:// orcid.org/0000-0001-7972-5247

Luis Cando Gaibor Md.

https:// orcid.org/0000-0001-7586-0097

Eder Chiriboga Dorado Md.

https:/ / orcid.org/0000-0002-7838-4724

Received: 06 - Jul - 2018

Accepted: 20 - Nov - 2019

Publish: 01 - Dec - 2019

Corresponding author: Walter Egas Ortega.

E-mail: walteregasortega@gmail.com

Authorship: Walter Egas Ortega conceptualized and designed the data, collected the data, analyzed and interpreted the data, did the statistical analysis and drafted the article. Walter Egas Romero analyzed and interpreted the data and drafted the article. Luis Cando Gaibor and Eder Chiriboga Dorado collected and analyzed the data. Julio Luzuriaga Graff conceptualized and designed the data, analyzed and interpreted the data, drafted the article, and critically revised the article.

Financial disclosure: The authors have no financial relationships relevant to this article to disclose.

STROBE 2008 Check List statement: The authors have real the STROBE 2008 Check List and the manuscript was prepared and revised according to the Strobe 2009 Checklist.

\section{Resumen}

La resolución quirúrgica de la hiperplasia prostática benigna se modifica conforme el acceso a las diferentes tecnologías en salud. Las complicaciones del abordaje endoscópico versus el convencional son similares. El Objetivo de este estudio fue analizar retrospectivamente los resultados de la cirugía convencional y la resección endoscópica monopolar de la hiperplasia de próstata en un hospital terciario del Ecuador.

Pacientes y Métodos: Estudio retrospectivo, analítico. Fueron incluidos en el estudio 232 pacientes con diagnóstico histopatológico de hiperplasia prostática benigna atendidos en el servicio de urología del hospital Luis Vernaza en el período enero 2015 - diciembre 2016. Los pacientes fueron divididos en 2 subgrupos de acuerdo con el abordaje terapéutico - quirúrgico: prostatectomía convencional ( $n=120$ ) y resección endoscópica $(n=112)$.

Resultados: Mediante estadística inferencial se comprobó una relación estadísticamente significativa entre el tipo de abordaje terapéutico con el tiempo quirúrgico ( $p=<0.001)$ y con el índice de sangrado $(p=<0.001)$ y entre la edad y el tiempo quirúrgico ( $p=<0.001)$. Las complicaciones más importantes fueron: hemorragia inmediata $(n=9)$ e infecciones de la herida ( $n=10$ ) para la cirugía convencional y hemorragia inmediata $(n=2)$ y sepsis $(n=3)$ para la cirugía endoscópica. La media de tiempo quirúrgico fue 103.03 min y 75.14 min respectivamente.

Conclusión: El desarrollo tecnológico ha traído la certeza de que la cirugía endoscópica tiene menores complicaciones, disminuye los tiempos quirúrgicos. Siendo una opción por considerar frente a la cirugía tradicional para la hiperplasia de próstata.

Palabras clave: próstata, hematuria, Prostatectomía, Resección Transuretral de la Próstata, fistula urinaria
Citation: Egas WD, Luzuriaga J, Sánchez $\mathrm{O}$, Egas WE, Cando L, Chiriboga E. Prostatectomía convencional vs resección endoscópica de próstata monopolar en pacientes con Hiperplasia Prostática: un estudio de corte retrospectivo. Rev Med Vozandes. 2019; 30 (2): 27 - 33 
Keywords: FALTAN KEYWORDS

\section{Abstract}

\section{Conventional prostatectomy vs. endoscopic resection of monopolar prostate in patients with Prostatic Hyperplasia: a retrospective study.}

Surgical resolution of benign prostatic hyperplasia is modified as access to different health technologies. Complications of endoscopic versus conventional approach are similar. The objective of this study was to retrospectively analyze the results of conventional surgery and monopolistic endoscopic resection of prostate hyperplasia in a tertiary hospital in Ecuador.

Patients and Methods: Retrospective, analytical study. 232 patients diagnosed with benign prostatic hyperplasia were included in the study in the urology service of the Luis Vernaza hospital in the period January 2015 - December 2016. Patients were divided into 2 subgroups according to the therapeutic-surgical approach: conventional prostatectomy (n.120) and endoscopic resection (n. 112).

Results: By inferential statistics, a statistically significant relationship was found between the type of therapeutic approach with the surgical time (p. 0.001) and with the bleeding index (p. 0.001) and between age and surgical time (p. 0.001). The most important complications were: immediate bleeding (n-9) and wound infections (no. 10) for conventional surgery and immediate bleeding (n-2) and sepsis (no. 3) for endoscopic surgery. The surgical mean time was $103.03 \mathrm{~min}$ and 75.14 min respectively.

Conclusions: Technological development has brought the certainty that endoscopic surgery has fewer complications, decreases surgical times. Being an option to consider against traditional prostate hyperplasia surgery.

\section{Introducción}

La hiperplasia prostática benigna, es el tumor más frecuente en adultos del sexo masculino, su incidencia aumenta con la edad y se asocia a síntomas urinarios bajos a partir de los 40 años. ${ }^{(1,2)}$ En el 2016 representó la séptima causa de morbilidad masculina en el Ecuador con 6615 casos (1.64\%). En el mismo año la provincia del Guayas - Ecuador, reportó 961 (0.36\%) egresos, con un promedio de 4.83 días de estancia hospitalaria. (3) Su tratamiento quirúrgico sigue siendo bimodal: a) cirugía convencional que no ha podido ser reemplazada con el tiempo y b) cirugía mínimamente invasiva - resección endoscópica de la próstata. ${ }^{(4)}$ La prostatectomía abierta ofrece tasas de retratamiento más bajas, resección completa del adenoma prostático bajo visión directa y ausencia de hiponatremia dilucional (síndrome de resección transuretral de la próstata), no obstante, sus principales limitantes son las complicaciones relacionadas a la herida 
quirúrgica - mayor tiempo de internación, convalecencia más prolongada y mayor ausentismo laboral. $(5,6)$ Por otro lado las técnicas endoscópicas visan disminuir estas complicaciones permitiendo una recuperación más rápida y un impacto económico menor. ${ }^{(5,6)}$

La edad avanzada, comorbilidades y los grandes volúmenes prostáticos al momento del diagnóstico, dificultan las decisiones terapéuticas. Estas disyuntivas motivaron este estudio con el objetivo de analizar retrospectivamente los resultados de la cirugía convencional y la resección endoscópica monopolar de la hiperplasia de próstata en un hospital terciario del Ecuador.

\section{Pacientes y métodos}

Esté fue un estudio de corte retrospectiva, fueron elegibles para el estudio pacientes adultos (>18 años) atendidos en los servicios de: Urología, Medicina Interna o Emergencia del Hospital Luis Vernaza de la ciudad de Guayaquil - Ecuador, con diagnóstico histopatológico de hiperplasia prostática benigna entre el 1 de enero del 2015 y el 31 de diciembre del 2016.

Pacientes que solicitaron alta voluntaria, referidos a otras instituciones, con prontuarios incompletos, con diagnóstico histopatológico diferente a hiperplasia prostática benigna o con resultados histopatológicos externos a la institución fueron excluidos.

Este estudio sigue las directrices STROBE para estudios observacionales y fue aprobado por el Comité de Ética del Hospital Luis Vernaza. El consentimiento informado no fue necesario debido al diseño no intervencionista y al carácter retrospectivo del estudio.

Datos referentes a: a) variables demográficas y clínicas, b) relacionados con la patología prostática de base: diámetro, volumen, estudio histopatológico y c) tratamiento: tipo de abordaje (endoscópico o convencional), tiempo quirúrgico, volumen de sangrado, tiempo de sonda, tiempo de irrigación, tiempo de internación hospitalaria y complicaciones fueron obtenidos directamente del prontuario de cada paciente.

La posibilidad de un abordaje convencional - prostatectomía abierta-está indicado en los casos en los que el tejido prostático pesa más de $75 \mathrm{~g}$, inseguridad del cirujano para resecar grandes volúmenes o la ausencia de recurso tecnológicos para realizar resecciones endoscópicas. ${ }^{(7)}$ Las principales indicaciones de la prostatectomía suprapúbica son: a) presencia de cálculos de gran tamaño en la vejiga, b) divertículos vesicales y c) lóbulo prostático medio voluminoso que protruye en la vejiga. ${ }^{\left({ }^{8}\right)}$

Por otro lado, las indicaciones del abordaje endoscópico representan: a) la retención recurrente de orina, b) macrohematuria refractaria a terapias con 5 alfa-reductasa, c) insuficiencia renal y d) dilatación del árbol urinario superior. (9)

El postoperatorio de la resección endoscópica se caracteriza por la presencia de hematuria, que en algunas ocasiones puede obstruir el catéter vesical. El lavado con soluciones estériles lo corregirá en la mayoría de las veces. En otras, será necesario cambiar el catéter in situ o en el quirófano. En casos de sangrado incontrolable es necesario realizar un empaquetamiento quirúrgico con gasa de Mikulicz en la celda prostática. $(10,11)$

Todos los pacientes recibieron anestesia peridural ${ }^{12)}$. La cirugía transvesical - técnica de Freyer, es más laboriosa, porque implica una cistotomía amplia con cierre en 2 planos con puntos continuos. Sus principales indicaciones son: presencia concomitante de tumores vesicales, cálculos, divertículos y portadores de hernias inguinales que se pretenda resolver por la pared interna. ${ }^{(13)}$ Su principal desventaja es la visión deficiente de la totalidad de la fosa prostática después de la enucleación. (14) La prostatectomía retropúbica de Millin se la realiza en pacientes sin lesiones intravesicales y sin infección urinaria. Su principal ventaja radica en la posibilidad de lograr la curación definitiva con micción espontanea sin esfuerzo, en un solo tiempo. Su principal desventaja es la dificultad para inspeccionar la cavidad vesical. ${ }^{(15)}$

La resección endoscópica fue realizada de acuerdo con la técnica de Mavermeyer comenzando entre 5' y 7' del horario cistoscópico (16).

La totalidad de los casos utilizaron agua estéril para irrigación, Baxter ${ }^{\circledR}$ durante el trans y posoperatorio. Siendo la hemostasia de la celda prostática un factor trascendente para la continuidad de la irrigación vesical. Las soluciones de irrigación tienen efectos adversos dependiendo de su composición. (17)

\section{Análisis estadístico}

Para el análisis estadístico fue utilizado IBM SPSS software package, version 22.0 for Windows (IBM, Armonk, NY, USA). Las características de los pacientes se expresaron como media y desviación estándar para variables continuas y recuentos y porcentajes para variables categóricas. La comparación entre grupos se realizó utilizando la prueba T de student o la prueba de MannWhitney para variables continuas. Las variables categóricas se compararon utilizando la prueba de chi-cuadrado de Pearson o la prueba exacta de Fisher según corresponda. Valores $p<0,05$ se consideraron estadísticamente significativos.

\section{Resultados}

Entre 1 de enero del 2015 y 31 de diciembre del 2016, fueron evaluados 290 pacientes 
con sintomatología urinaria baja y diagnóstico clínico de hiperplasia prostática benigna. De estos, 58 (20\%) fueron excluidos: 5 (1.72\%) por alta voluntaria, 5 (1.72\%) referidos a otros hospitales, $15(5.17 \%)$ por resultados histopatológicos diferentes a hiperplasia prostática benigna y 33(11.37\%) por resultados histopatológicos externos a la institución. Los 232 pacientes que conforman nuestra muestra fueron divididos en dos subgrupos de acuerdo con la modalidad quirúrgica usada: a) prostatectomía convencional, y b) resección endoscópica monopolar. (Tabla 1).

Se comprobó una relación estadísticamente significativa entre la modalidad quirúrgica/tiempo quirúrgico ( $p<0.001)$, modalidad quirúrgica/índice de sangrado (<0.001), edad/ tiempo quirúrgico $(<0.001)$. No se encontró una asociación estadísticamente significativa entre las variables hospitalización/ modalidad quirúrgica ( $p=0.198)$ y tiempo de hospitalización/ modalidad quirúrgica $(p=0.172)$.

\section{Discusión}

La relación positiva o negativa de las variables involucradas en un evento quirúrgico de próstata siempre ha sido transmitida en el servicio como una anécdota coloquial sin embargo la opinión de un experto tiene bajo nivel de evidencia en cualquier guía de práctica clínica.

Estudio realizado en la ciudad de Lima por Espinoza $\mathrm{E}$, et al. (18), con una muestra de 150 pacientes, reportó una media de 150 minutos para la cirugía retropúbica ${ }^{(18)}$. El trabajo de Acuña JA, et al. ${ }^{(19)}$, con una muestra de 15 pacientes de un hospital

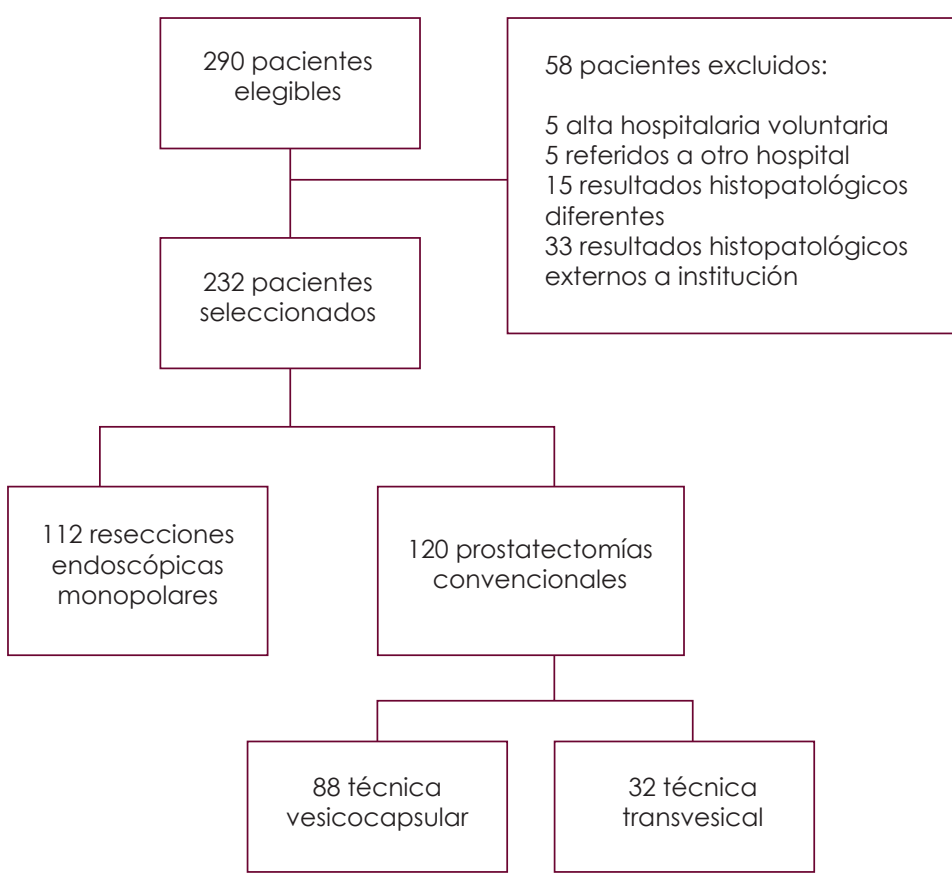

Fuente: el autor/prontuario de los pacientes.

Figura 1. Flujograma de pacientes elegibles e incluidos en el análisis, categorizados de acuerdo con la modalidad quirúrgica (enero 2015 - diciembre 2016) docente de Monterey, encontró una media de tiempo quirúrgico de 61 minutos (19). A pesar de que las tres muestras presenten características semejantes, la media de tiempo quirúrgico menor en nuestra serie probablemente refleje la experiencia de nuestro servicio.

La próstata hiperplásica es altamente vascularizada y el sangrado transoperatorio usualmente es significativo sin embargo en resecciones endoscópicas estimar su volumen es inexacto y difícil, porque la sangre se mezcla con el fluido de irrigación. ${ }^{(20)}$ Se estima que las pérdidas varíen de acuerdo con el tiempo de resección (2 - $5 \mathrm{ml}$ por minuto de resección) y con el peso de la glándula $(20-50 \mathrm{ml}$ por gramo de tejido). (21)

De acuerdo con los resultados de Kirollos MM, et al. $\left.{ }^{(22}\right)$, los factores asociados con mayor pérdida sanguínea fueron: el peso del tejido prostático resecado, tiempo de resección, anestesia general y la necesidad de cirugía urgente para resecciones endoscópicas.

Para resecciones convencionales los factores asociados fueron: técnica de Freyer, edad mayor de 70 años, uso de acetilcisteína o warfarina perioperatorias, presión arterial sistólica > 140 mmHg, anestesia general y volumen resecado mayor a 70 gramos. (23)

En nuestra casuística no hubo relación entre el peso del volumen prostático y el índice de sangrado, por otro lado, considerando el volumen de sangrado por el tiempo de resección podríamos inferir que nuestros pacientes tuvieron una pérdida permisible de $335 \mathrm{ml}$, sin embargo, estos cálculos son imprecisos.

La tecnología de resección monopolar está asociada con hiponatremia debido a que utiliza un sistema de irrigación no conductivo con agua estéril, solución isotónica/iso-osmolar, con respecto al plasma humano. ${ }^{(14)}$ El síndrome post-resección de próstata tiene una incidencia en la literatura del $1 \%$ al $3 \%$ caracterizado por confusión mental, nausea, vomito, hipertensión, bradicardia y alteraciones visuales. Es causado por hiponatremia dilucional (sodio sérico $<125$ $\mathrm{mEq} / \mathrm{ll}$ debido a la perforación de venas o sinusoides de la capsula con el consiguiente flujo de solución de irrigación hipotónica. (24) Puede manifestarse en forma de alteraciones neurológicas (92\%), cardiovasculares (54\%), alteraciones visuales (42\%) y digestivas (25\%). Sin embargo, al carecer de una presentación clínica definida es difícil precisar su incidencia real. $(24,25)$ En nuestro servicio no se realizan ionogramas como parte del protocolo rutinero de cuidados postoperatorios a menos que exista algún tipo de deterioro clínico o complicaciones, en los prontuarios analizados no se encontraron 


\section{ARTÍCULO ORIGINAL Estudio retrospectivo}

Tabla 1 - Características demográficas, clínicas y datos quirúrgicos de los 232 pacientes incluidos en el estudio categorizados por tipo de abordaje quirúrgico

\begin{tabular}{|c|c|c|}
\hline Características de los pacientes & $\begin{array}{c}\text { Prostatectomía } \\
\text { convencional } \\
n=120\end{array}$ & $\begin{array}{c}\text { Resección } \\
\text { endoscópica de } \\
\text { próstata } n=112\end{array}$ \\
\hline Edad (años) $\pm S D$ & $71.14 \pm 9.30$ & $69.67 \pm 9.53$ \\
\hline \multicolumn{3}{|l|}{ Comorbilidades, no (\%) } \\
\hline Hipertensión & $26(21.60)$ & $22(19.64)$ \\
\hline Diabetes Mellitus & $11(9.16)$ & $14(12.50)$ \\
\hline Cardiopatías & $3(2.50)$ & $4(3.57)$ \\
\hline Insuficiencia renal & $10(8.33)$ & $7(6.25)$ \\
\hline Neurológicos & $8(6.66)$ & $4(.57)$ \\
\hline Otros & $18(15.00)$ & $13(11.60)$ \\
\hline \multicolumn{3}{|l|}{ Cirugías previas, no (\%) } \\
\hline Urología & $11(9.16)$ & $7(6.25)$ \\
\hline Digestivas & $1(0.83)$ & $4(3.57)$ \\
\hline Otras & $2(1.66)$ & $0(0.00)$ \\
\hline \multicolumn{3}{|l|}{ Relacionadas con la cirugía } \\
\hline Tiempo quirúrgico (min) $\pm S D$ & $103.03 \pm 34.83$ & $75.14 \pm 30.62$ \\
\hline Sangrado $(\mathrm{ml}) \pm S D$ & $296.33 \pm 197.76$ & $105.67 \pm 106.324$ \\
\hline Volumen Prostático (gr)士SD & $67.22 \pm 37.87$ & $20.84 \pm 17.74$ \\
\hline Tiempo irrigación (días) „SD & $3.47 \pm 1.2$ & $2.93 \pm 0.96$ \\
\hline Tiempo de sonda (días) $\pm S D$ & $6.81 \pm 1.97$ & $4.30 \pm 1.56$ \\
\hline Tiempo internación (días) „SD & $16.68 \pm 20.78$ & $13.27 \pm 19.28$ \\
\hline \multicolumn{3}{|c|}{ Complicaciones inmediatas (hasta 24 horas) no (\%) } \\
\hline Hemorragia inmediata & $9(7.50)$ & $2(1.78)$ \\
\hline Retención completa de orina por sangrado & $2(1.66)$ & $2(1.78)$ \\
\hline Choque hipovolémico III-IV & $2(1.66)$ & $0(0.00)$ \\
\hline Taponamiento de celda prostática & $4(3.33)$ & $0(0.00)$ \\
\hline \multicolumn{3}{|c|}{ Complicaciones mediatas (mayor de 24 horas hasta 30 días) no (\%) } \\
\hline Seroma & $4(3.33)$ & $0(0.00)$ \\
\hline Infección de la herida & $10(8.33)$ & $0(0.00)$ \\
\hline Dehiscencia de sutura & $1(0.83)$ & $0(0.00)$ \\
\hline Fistula vesico- cutánea & $9(7.50)$ & $0(0.00)$ \\
\hline Epidídimo-orquitis & $1(0.83)$ & $1(0.89)$ \\
\hline Sepsis de foco urinario & $6(5.00)$ & $3(2.67)$ \\
\hline Choque séptico & $0(0.00)$ & $1(0.89)$ \\
\hline neumonía & $3(2.50)$ & $1(0.89)$ \\
\hline Gangrena de Fournier & $1(0.83)$ & $0(0.00)$ \\
\hline Hematuria grave & $4(3.33)$ & $1(0.89)$ \\
\hline Retención completa de orinas por sangrado & $4(3.33)$ & $1(0.89)$ \\
\hline Incontinencia urinaria & $2(1.66)$ & $0(0.00)$ \\
\hline Atonía vesical por retiro de catéter & $0(0.83)$ & $10(8.92)$ \\
\hline \multicolumn{3}{|c|}{ Complicaciones tardías (mayor de 30 días) no. (\%) } \\
\hline Estenosis de uretra & $1(1.66)$ & $1(0.89)$ \\
\hline Tejido prostático residual & $0(0.83)$ & $1(0.89)$ \\
\hline Muerte & $1(1.66)$ & $0(0.00)$ \\
\hline $\begin{array}{l}\text { Datos expresados como medias } \pm \text { SD. } \\
\text { SD: Desviación estándar. }\end{array}$ & & \\
\hline
\end{tabular}


hallazgos clínicos que sugieran síndrome post resección endoscópica. La sonda vesical tutoriza el tracto urinario inferior para permitir la cicatrización de la celda prostática, y se debería mantener hasta que ceda por completo la hematuria, siendo la principal indicación en nuestro servicio. Otra indicación es la presencia de orinas claras luego de 24 horas de retirado el sistema de irrigación. Evitando de esta forma la retención de orinas por coágulos. ${ }^{(26,27)}$

Existen diversas soluciones empleadas para irrigación, soluciones a base de manitol causan retención de volumen en el espacio vascular con la consiguiente hipovolemia y hemodilución y posibilidad de insuficiencia cardiaca en un paciente geriátrico. ${ }^{(28)}$ La glicina causa aumento de los niveles de amonio no obstante se necesitan aumentos de 10-15 veces para causar encefalopatía. ${ }^{(29)}$ El sorbitol se metaboliza en glucosa, fructuosa y lactato, produciendo un efecto osmótico similar e inferior al manitol y actualmente se considera la solución con menos efectos adversos. ${ }^{\left({ }^{30}\right)}$ Nosotros no encontramos ningún caso de hiponatremia dilucional, comparado con un $6.46 \%$ en una muestra de 21 resecciones, estudiadas por Arbej JP, et al.(6) La fistula vesico-cutánea es la mayor y más grave complicación que puede ocurrir en el sitio quirúrgico. Una sutura de la pared vesical que deje los bordes evertidos produce un ectropión de la mucosa dando lugar a una cicatriz hipertrófica que se puede adherir a los planos más superficiales (musculo, fascia) originando una eventración y la consiguiente fistula, la misma que se encuentra asociada con mayor frecuencia a la técnica transvesical. ${ }^{(31,32)}$ Datos sobre este tipo de fístulas son escasos, Lloontop J, et al. ${ }^{(33)}$, en una muestra de 161 pacientes, operados con esta técnica, describen una prevalencia de $16.7 \%$ de fistulas vesicocutaneas ${ }^{(33)}$. En nuestro estudio encontramos una prevalencia de $7.5 \%$ entre ambas modalidades terapéuticas, su desarrollo depende de la técnica empleada, la destreza del cirujano, nutrición del paciente y la presencia de colonización bacteriana en la orina.

La próstata es puerto de muchas bacterias, pudiendo causar bacteriemia intraoperatoria o postoperatoria. El riesgo aumenta por la presencia del catéter vesical permanente. Es causada por bacterias gram positivas y gram negativas. Tiene una incidencia del $6 \%$ al $7 \%$. ${ }^{(20)}$ El principal factor para la infección del sitio quirúrgico en Urología es la presencia de infección urinaria previa a la cirugía. La orina infectada contamina los campos quirúrgicos y luego la herida quirúrgica. (20) En una revisión de complicaciones en cirugía urológica, realizadas en Zaragoza, por Arbej JP, et al. (6), en una muestra de 21 pacientes encontraron 19 infecciones de sitio quirúrgico en cirugías de adenomectomía de próstata. ${ }^{(35)}$ La epidídimoorquitis y la neumonía también se explica por esos procesos fisiopatológicos. La mortalidad asociada a sepsis urinaria es del 25 al $75 \%$. (30)

La profilaxis antibiótica es una medida eficaz que disminuye la frecuencia de infecciones bacterianas postquirúrgicas. ${ }^{\left({ }^{34}\right)}$ En cirugía urológica se han realizado numerosos estudios comparando profilaxis antimicrobiana de corta versus larga duración, no obstante, el uso prolongado no se asoció a mejores resultados por lo tanto la Asociación Europea de Urología recomienda una sola dosis preoperatoria de profilaxis para cirugías limpia-contaminadas. ${ }^{(35)}$

La principal limitación de este estudio son las relacionadas con la naturaleza retrospectiva y realizada en un único centro. La falta de una muestra homogénea para la prostatectomía transvesical (32 vs 88 retropúbica) impidió que se realicen análisis entre estas dos técnicas convencionales y entre el abordaje endoscópico monopolar. La ausencia de otras tecnologías para la resección endoscópica de próstata, disminuyen las probabilidades de hacer estudios observacionales sobre cirugía mínimamente invasiva de patología benigna de esta glándula.

En conclusión, siendo tantos los factores involucrados en un acto quirúrgico y sus resultados posteriores, no puede precisarse si la realidad percibida puede extrapolarse a otros grupos, sin embargo la selección correcta de pacientes para cada uno de los abordajes reducirá el número de complicaciones, por otro lado el abordaje endoscópico parecía estar asociado con mejores resultados, sin embargo hacen falta más estudios para poder establecer un gold estándar en el tratamiento de esta patología.

\section{Agradecimiento}

En memoria del Dr. Oscar Sánchez Chávez†, sin cuya escuela no se hubieran podido obtener el beneficio para los pacientes atendidos en el servicio de Urología del Hospital Luis Vernaza.

John Rivera, estudiante universitario, que colabora con la revisión del abstracto en inglés.

\section{Presentaciones previas}

Este estudio fue presentado en formato de tema libre durante XXXVI Congreso de la Confederación Americana de Urología en Santa Cruz - Bolivia, el 18 de octubre del 2017, en el XXXV Congreso Nacional de Urología en la ciudad de Loja - Ecuador, el 17 de noviembre de 2017 y en el XLI Congreso Nacional de Cirugía, en Guayaquil - Ecuador, el 16 de mayo del 2018. 


\section{Referencias}

1. Serretta V, Morgia G, Fondacaro L, Curto G, Lo bianco A, Pirritano D, et al. Open prostatectomy for benign prostatic enlargement in southern Europe in the late 1990s: a contemporary series of 1800 interventions. Urology. 2002 Oct $; 60(4): 623-7$

2. Fernández C, Moreno J, Cano S, Fuentes M.E Bocardo G, Silmi Á. Prevalencia de síntomas del tracto urinario inferior relacionados con la hiperplasia benigna de próstata: Estudio de 1804 hombres de 40 años o más residentes en Madrid durante 1999-2000. Actas Urología Española. 2009 Ene; 33(1):43-51.

3. Villacís B, Carrillo D. Estadística Demográfica en el Ecuador: Diagnóstico y Propuesta [Hompage en Internet]. Quito: Instituto. Nacional de Estadística y Censos (INEC). 2012; Ener. 1 (1):35 38 [Citado 2017 Dic 23]. Disponible en: https:// www.ecuadorencifras.gob.ec/wp-content/ descargas/Libros/Demografia/documentofinall.pdf

4. Fitzpatrick J. Tratamiento mínimamente invasivo y endoscópico de la hiperplasia prostática benigna. "In" Campbell W. Urología. 2007; 2804 2844.

5. Roehrborn C, McConnell J. Hiperplasia prostática benigna de próstata: etiología, fisiopatología, epidemiología y evolución natural. "In" Campbell W. Urología. 2007; 2727-2803.

6. Arbej JP, Rico MC, Cameo CP, Doñate RM. Infección de herida quirúrgica en pacientes urológicos. Revisión de los casos registrados en cuatro años. Actas Urológicas Españolas. 2009; $34(3): 258-265$.

7. Suer E, Gokce I, Yaman O, Anafarta K, Gögüs $O$. Open prostatectomy is still a valid option for large prostates: a high-volume, single-center experience. Urology. 2008 Jul;72(1):90-4. doi: 10.1016/j.urology.2008.03.015.

8. Gratzke C, Schlenker B, Seitz M, Karl A, Hermanek $P$, Lack $N$, et al. Complications and early postoperative outcome after open prostatectomy in patients with benign prostatic enlargement: results of a prospective multicenter study. J Urol. 2007 Apr;177(4):1419-22.

9. Marszalek M, Ponholzer A, Pusman M, Berger I, Madersbacher S. Transurethral resection of the prostate. European urology supplements. 2009:8(6):504-512.

10. Hubmann R. Geschichte der transurethralen Prostataeingriffe. Der Urologe B. 2000; 40(2) 152-160.

11. Valdivia GG, Arellano SM. Bosquejo histórico de los drenajes en cirugía y sus autores. Cir Gen. 2016; 38(1):35-40.

12. McGowan SW, Smith GF. Anaesthesia for transurethral prostatectomy. A comparison of spinal intradural analgesia with two methods of general anaesthesia. Anaesthesia. 1980 Sep;35(9):847-53.
13. Raspall P. Superioridad de la vía retropúbica, en la técnica de la prostatectomía (o adenomectomía). Estadística y comentarios sobre 47 casos intervenidos. Anales de medicina y cirugía. 1949;26(51):197-209.

14. Condie JD Jr, Cutherell L, Mian A. Suprapubic prostatectomy for benign prostatic hyperplasia in rural Asia: 200 consecutive cases. Urology. 1999 Dec;54(6):1012-6.

15. Martínez FM, Pérez PA. Prostatectomía según la técnica de Freyer en dos tiempos: recuerdo histórico. ENFURO: Asociación Española de Enfermería en Urología. 1995; (54): 47-52.

16. Hubmann R, Opelt B. [Conservative and surgical management of prostatic diseases]. Internist (Berl). 1968 May;9(5):207-13.

17. Ramos L, Rueda F, Sancho A, Esteban J, Cajigal I, Fabregat $C$, et al. Síndrome de reabsorción post-resección transuretral (RTU) de próstata: revisión de aspectos fisiopatológicos, diagnósticos y terapéuticos. Actas urología española. ticos y terapeuticos.
$2001 ; 25(1): 14-31$.

18. Espinoza E. Estudio comparativo de los resultados de las técnicas quirúrgicas de adenomectomía prostática retropúbica y adenomectomía prostática transvesical en el servicio de Urología del Hospital Nacional servicio de Urología del Hospital Nacional Lima: Universidad Ricardo Palma; 2009.

19. Acuña JA, Hernández AU, Gómez LS, Martínez R, Lozano JF. Resección transuretral de próstata bipolar vs monopolar. Análisis peri y posoperatorio de los resultados. Revista Mexicana de Urología. 2010 May;70(3): 146-151.

20. Pientka L, van Loghem J, Hahn E, Guess H, Keil U. Comorbidities and perioperative complications among patients with surgically treated benign prostatic hyperplasia. Urology. 1991;38(1 Suppl):43-8.

21. Friedman NJ, Hoag MS, Robinson AJ, Aggele PM. Hemorrhagic syndrome following transurethral prostatic resection for benign adenoma. Arch Intern Med. 1969Sep; 124(3):341-9.

22. Kirollos MM, Campbell N. Factors influencing blood loss in transurethral resection of the prostate (TURP): auditing TURP. Br J Urol. 1997 Jul;80(1):111-5.

23. Heathcote PS, Dyer PM. The effect of warm irrigation on blood loss during transurethral prostatectomy under spinal anaesthesia. Br J Urol. 1986 Dec;58(6):669-71.

24. Ramos LC, Almagro AA. Síndrome de reabsorción post-resección transuretral (RTU) de próstata: revisión de aspectos fisiopatológicos, diagnósticos y terapéuticos. Actas Urología diagnósticos y terapéuticos. A
Española. 2001 Jan;25(1): 14-31.

25. Kolmert T, Norlén H. Transurethral resection of the prostate. A review of 1111 cases. Int Uro Nephrol. 1989;21 (1):47-55.
26. Norlén H. Isotonic solutions of mannitol, sorbitol and glycine and distilled water as irrigating fluids during transurethral resection of the prostate and calculation of irrigating fluid influx. Scand J Urol Nephrol Suppl. 1985; 96:1-50,81p.

27. Hoekstra PT, Kahnoski R, McCamish MA, Bergen W, Heetderks DR. Transurethral prostatic resection syndrome--a new perspective: encephalopathy with associated hyperammo nemia. J Urol. 1983 Oct;130(4):704-7.

28. Zheng $X$, Han $X$, Cao D, Wang $Y, X u H$, Yang $L$, et al. Comparison of Short-Term Outcomes between Button-Type Bipolar Plasma Vaporization and Transurethral Resection for the Prostate: A Systematic Review and Meta-Analysis. Int J Med Sci. 2019 Oct 21;16(12):1564-1572. doi: 10.7150/ijms.38618.

29. Betschart P, Pratsinis M, Müllhaupt $G$, Rechner $R$, Herrmann TR, Gratzke C,et al. Information on surgical treatment of benign prostatic hyperplasia on YouTube is highly biased and myperplasia on YouTube is highly biased and misleading.
bju.14971.

30. Lokeshwar SD, Harper BT, Webb E, Jordan A Dykes TA, Neal DE Jr, et al. Epidemiology and treatment modalities for the management of benign prostatic hyperplasia. Transl Androl Urol. 2019 Oct:8(5):529-539. doi: 10.21037/ tau.2019.10.01. Review.

31. Simpson P. Prostatectomy: information pro vision and education for patients. $\mathrm{Br} \mathrm{J}$ Nurs. 2015 May 14-27;24(9):S24-8. doi: 10.12968/ bjon.2015.24.Sup9.S24.

32. Rodríguez F, Vidal R, Burgos J, Soto M. Fístulas vesicocutáneas y genitales. Clínicas Urológicas de la Complutense. 1998; (6):441-453.

33. Lloontop J, Ludeña R. Complicaciones postoperatorias de la Hiperplasia benigna de próstata en el Hospital Luis Heysen Inchaustegui durante el período enero 2012 -diciembre 2014. Chiclayo: Universidad Nacional Pedro 2014. Chiclayo:
Ruiz Gallo. 2015.

34. Lepor H, Nieder AM, Ferrandino MN. Intrao perative and postoperative complications of radical retropubic prostatectomy in a consecutive series of 1,000 cases. J Urol. 2001 Nov;166(5):1729-33.

35. Sakura M, Kawakami S, Yoshida S, Masuda $\mathrm{H}$, Kobayashi T, Kihara K. Prospective comparative study of single dose versus 3-day administration of antimicrobial prophylaxis in minimum incision endoscopic radical prostatectomy. Int J Urol. 2008 Apr; 15(4):328-31. doi: 10.1111/j.1442-2042.2008.02001.x. 